\title{
OPEN High-throughput quantitation of SARS-CoV-2 antibodies in a single-dilution homogeneous assay
}

Markus H. Kainulainen ${ }^{1}$, Eric Bergeron ${ }^{1}$, Payel Chatterjee ${ }^{1}$, Asheley P. Chapman ${ }^{2}$, Joo Lee ${ }^{3}$, Asiya Chida ${ }^{3}$, Xiaoling Tang ${ }^{3}$, Rebekah E. Wharton ${ }^{4}$, Kristina B. Mercer ${ }^{5}$, Marla Petway ${ }^{3}$, Harley M. Jenks ${ }^{1}$, Timothy D. Flietstra' ${ }^{1}$, Amy J. Schuh ${ }^{1}$, Panayampalli S. Satheshkumar ${ }^{6}$, Jasmine M. Chaitram ${ }^{7}$, S. Michele Owen ${ }^{8}$, Laura K. McMullan' ${ }^{1}$, Mike Flint ${ }^{1}$, M. G. Finn', Jason M. Goldstein ${ }^{3}$, Joel M. Montgomery ${ }^{1}$ \& Christina F. Spiropoulou ${ }^{1 \bowtie}$

SARS-CoV-2 emerged in late 2019 and has since spread around the world, causing a pandemic of the respiratory disease COVID-19. Detecting antibodies against the virus is an essential tool for tracking infections and developing vaccines. Such tests, primarily utilizing the enzyme-linked immunosorbent assay (ELISA) principle, can be either qualitative (reporting positive/negative results) or quantitative (reporting a value representing the quantity of specific antibodies). Quantitation is vital for determining stability or decline of antibody titers in convalescence, efficacy of different vaccination regimens, and detection of asymptomatic infections. Quantitation typically requires twostep ELISA testing, in which samples are first screened in a qualitative assay and positive samples are subsequently analyzed as a dilution series. To overcome the throughput limitations of this approach, we developed a simpler and faster system that is highly automatable and achieves quantitation in a single-dilution screening format with sensitivity and specificity comparable to those of ELISA.

Detecting viral nucleic acids, primarily by reverse-transcription quantitative polymerase chain reaction (RTqPCR), is the method of choice for diagnosing acute viral infections. However, diagnosing acute infections does not account for asymptomatic infections or bias resulting from availability of care or individuals not seeking care ${ }^{1}$. Therefore, serology plays an important part in completing the epidemiological picture when a new pathogen, such as severe acute respiratory syndrome coronavirus 2 (SARS-CoV-2 $)^{2,3}$, emerges. Analyzing humoral responses in convenience samples is a quick way to estimate how many individuals have already been exposed to a pathogen. On the other hand, designing community-level and special population studies offers the opportunity to solicit tailored participant information, such as symptom history, for correlation analyses ${ }^{1}$. Apart from surveys that aim to produce generalizable information, serology has also been used to link together individual COVID-19 clusters where nucleic acid evidence was absent ${ }^{4}$.

While the serological studies mentioned above could potentially be conducted with qualitative serology assays (which report presence or absence of SARS-CoV-2 antibodies), answering other pertinent questions requires quantitative determination of antibody levels. One timely question is the possibility of waning immunity to

\footnotetext{
${ }^{1}$ Viral Special Pathogens Branch, Division of High-Consequence Pathogens and Pathology, Centers for Disease Control and Prevention, 1600 Clifton Rd, Atlanta, GA 30329, USA. ${ }^{2}$ School of Chemistry and Biochemistry, School of Biological Sciences, Georgia Institute of Technology, 901 Atlantic Dr., Atlanta, GA 30332, USA. ${ }^{3}$ Reagent and Diagnostic Services Branch, Division of Scientific Resources, Centers for Disease Control and Prevention, 1600 Clifton Rd, Atlanta, GA 30329, USA. "Emergency Response Branch, Division of Laboratory Sciences, Centers for Disease Control and Prevention, 4770 Buford Hwy., Atlanta, GA 30341, USA. 5 Newborn Screening and Molecular Biology Branch, Division of Laboratory Sciences, Centers for Disease Control and Prevention, 4770 Buford Hwy., Atlanta, GA 30341, USA. ${ }^{6}$ Poxvirus and Rabies Branch, Division of High-Consequence Pathogens and Pathology, Centers for Disease Control and Prevention, 1600 Clifton Rd, Atlanta, GA 30329, USA. ${ }^{7}$ Division of Laboratory Systems, Centers for Disease Control and Prevention, 1600 Clifton Rd, Atlanta, GA 30329, USA. ${ }^{8}$ National Center for HIV/AIDS, Viral Hepatitis, STD, and TB Prevention, Centers for Disease Control and Prevention, 1600 Clifton Rd, Atlanta, GA 30329, USA. ${ }^{\boxplus}$ email: ccs8@cdc.gov
} 
A)

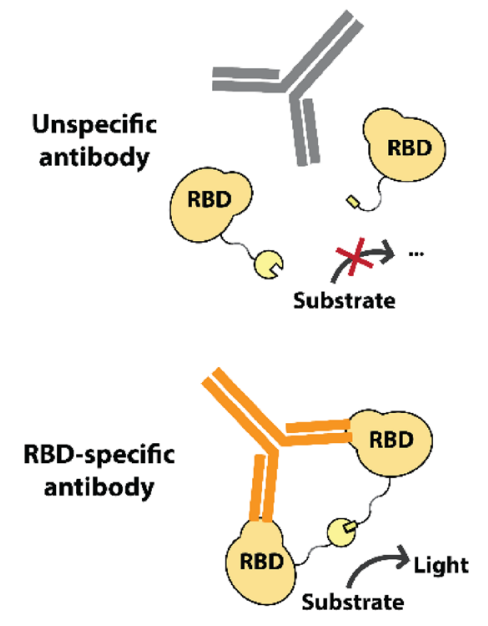

B)

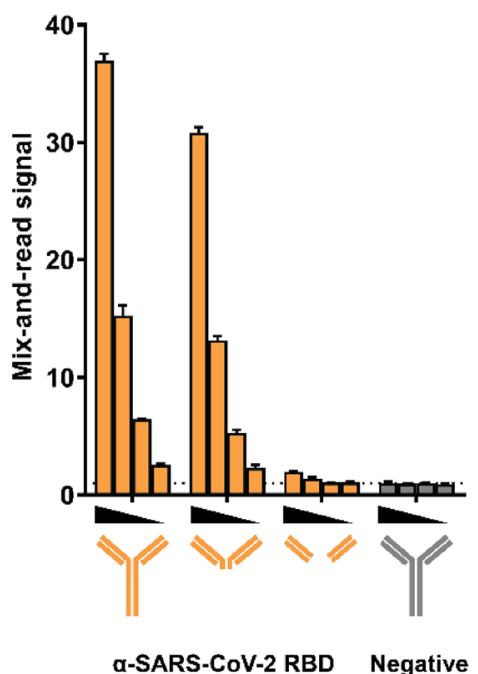

C)

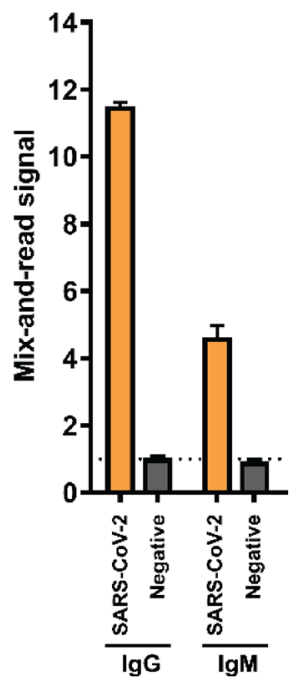

Figure 1. Assay principle. (A) Schematic. Small and large fragments of the NanoBit split luciferase are tethered to SARS-CoV-2 receptor-binding domains (RBD) via peptide linkers. When a specific antibody binds 2 RBD antigens, it forces the luciferase halves into proximity with each other, thereby activating the enzyme. Signal is quantified from a homogeneous assay setup after adding luciferase substrate. (B) Demonstration of assay principle. Dose-responsive assay activation by an RBD-specific monoclonal antibody or corresponding $\mathrm{F}\left(\mathrm{ab}^{\prime}\right)_{2}$ or Fab fragments (orange) or control antibody (gray). (C) Activation by specific IgG and IgM antibodies. Human IgG and IgM fractions were purified from a SARS-CoV-2 specific serum pool or a negative control pool and tested for activity in the assay. (B) and (C): technical triplicates with averages and standard deviations presented.

SARS-CoV-2 and reinfection with this pathogen. Some studies have called into question the long-term stability of the humoral immune response against this virus ${ }^{5-7}$, and small-scale human challenge studies with a benign human coronavirus have shown that at least asymptomatic reinfection with the same strain ${ }^{8}$ or a related one $e^{9}$ is possible as soon as a year after initial infection. Meanwhile, other studies did not observe rapid waning of the humoral response $\mathrm{e}^{10,11}$ and yet others have evaluated the immune signature of mild SARS-CoV-2 infections and predicted that this immune memory is likely to be protective ${ }^{12}$. Longitudinal sampling combined with accurate serological quantitation is necessary to monitor variations in antibody levels associated with waning immunity or reinfection ${ }^{13}$. Vaccine trials also require quantitative serology to establish immune correlates and to decide on optimal vaccine dose and regimen.

Another timely question is the role that other animal hosts may play in the future of the SARS-CoV-2 pandemic. To date, the natural reservoir or intermediate hosts leading to the emergence of this virus in humans have not been definitively identified. However, cats $^{14}$, deer mice ${ }^{15}$, ferrets, fruit bats ${ }^{16}$, and golden hamsters ${ }^{17,18} \mathrm{can}^{1}$ transmit the virus in a laboratory setting. Furthermore, SARS-CoV-2 outbreaks have occurred in farmed mink, and virus phylogenetic evidence suggest that mink may have transmitted the virus back to humans ${ }^{19}$. One-health investigations into the reservoir species and potential intermediate hosts of SARS-CoV-2, as well as into spillover events into species with which people have frequent contact, would benefit from species-independent serology methods to determine the role these species play in virus transmission and spread in human populations.

Protein complementation (also referred to as protein fragment complementation) is an assay principle primarily used for studying protein-protein interactions in cells. The interacting proteins are coupled to protein or peptide moieties that generate a detectable signal only when brought into close proximity with each other. Fluorescence-based complementation assays can provide information on protein-protein interactions and on the localization of the interaction in living cells. Such assays have been developed by dividing the structure of the green fluorescent protein and its relatives into fragments that only fluoresce when in close proximity. Luciferasebased systems, however, are generally more sensitive than fluorescent protein systems ${ }^{20,21}$. The recent such splitting of NanoLuc, the most sensitive luciferase to date $\mathrm{e}^{22}$, enabled us to build a serological assay that matches the sensitivity and specificity characteristics of classical enzyme-linked immunosorbent assays (ELISAs) but also offers easier automation, higher throughput, and independence of host species while quantitating antibodies in a single step.

\section{Results}

Assay principle. We hypothesized that antibodies, having two or more antigen binding sites (depending on class), could act as 'protein complementation bridges' to bring together antigens in solution, resulting in a detectable signal (Fig. 1A). The receptor-binding domain (RBD) of SARS-CoV-2 spike protein was chosen as the assay antigen, since it is known to offer high specificity against prevalent antibodies against common human coronaviruses $^{23,24}$. Two RBD-fusion proteins, each with one fragment of the split NanoBit luciferase ${ }^{22}$, were produced in mammalian cells and purified. To test if binding of both fragments simultaneously could indeed result 
A)

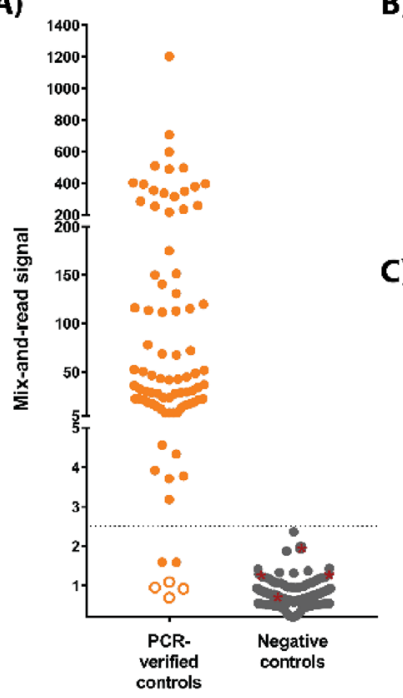

B)

C)
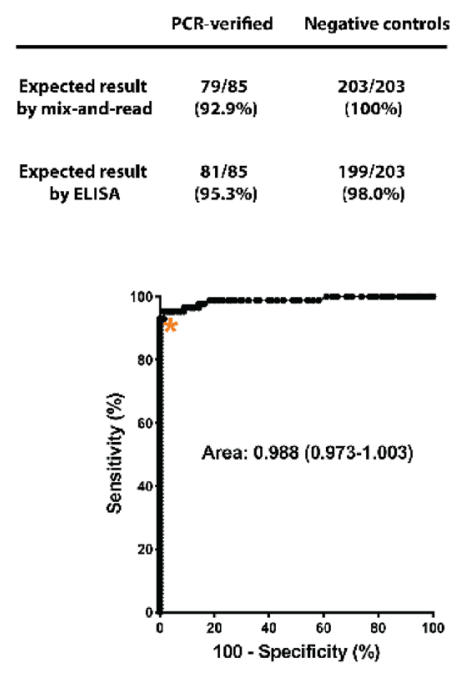

Figure 2. (A) Training set data. Serum samples from cases with earlier nucleic acid confirmed SARS-CoV-2 infection and samples considered true negatives (no COVID-19 diagnosis, or sample collected prior to SARSCoV-2 emergence) were analyzed by the mix-and-read assay and by RBD ELISA. Out of 85 positive controls, 79 were above proposed cut-offs on both ELISA and mix-and-read assays (orange symbols above signal 2.5), 4 gave no signal distinguishable from negative controls on either assay (open orange symbols), and 2 were positive on ELISA only (orange symbol below 2.5). All negative control signals $(\mathrm{N}=203$ ) were below proposed mix-andread cut-off (gray symbols), but 4 of those samples were positive by ELISA (gray symbols highlighted by red asterisks). (B) Summary of the training set data. (C) Receiver operating characteristics (ROC) analysis of the data and cut-off selection. Cut-off value of 2.5 (as fold over blank samples) was chosen by ROC analysis of the training set data so that $100 \%$ specificity could be maintained.

in protein complementation, we compared the signal generated by a monoclonal antibody ( $\mathrm{mAb}$ ) either in its intact form, or as $\mathrm{F}\left(\mathrm{ab}^{\prime}\right)_{2}$ or Fab fragments produced by protease digestion. Signal could be observed when intact $\mathrm{mAb}$ and $\mathrm{F}\left(\mathrm{ab}^{\prime}\right)_{2}$ were used, but not with Fab fragments, indicating that antibody binding two antigens simultaneously is necessary for assay activation (Fig. 1B). All three forms of the $\mathrm{mAb}$ retained the ability to inhibit interaction of RBD with its receptor, ACE2 (angiotensin-converting enzyme 2), showing that despite being unable to activate the assay the Fab fragment retained binding affinity for the RBD antigen (Supplementary Fig. 1A and B).

Since signal generation is dependent on an antibody binding two RBD antigens but does not require the $\mathrm{Fc}$ region, we next investigated whether purified antibodies from convalescent COVID-19 patients yield a signal and whether the pentameric structure of IgM antibodies could also activate the RBD reporter pair. Total IgG and IgM fractions were purified from a convalescent COVID-19 serum pool and a control pool (Supplementary Fig. 2). The system could be activated by the specific IgG fraction and the specific IgM fraction, indicating that the assay measures total immunoglobulin reactivity against the RBD (Fig. 1C).

Training set and cut-off determination. Assay performance was optimized by varying factors, including antigen concentrations and ratios, buffer and blocker choices, plastic materials, incubation times, and substrates. Additionally, we observed that using RBD domain consisting of SARS-CoV-2 residues $319-591^{25}$ offered lower background luminescence signal, and therefore higher sensitivity, than using residues 319-541 ${ }^{26}$ (optimization data not shown).

In order to establish the assay in human diagnostics, we opted to use the two-step strategy, in which the assay cut-off was first established with a training sample set, and performance was then validated with an independent sample set. The training set consisted of 288 serum samples: 85 samples from individuals with prior SARS-CoV- 2 infection confirmed by nucleic acid testing, and 203 negative samples (117 negative control samples sourced for the purpose, 100 of which were collected prior to SARS-CoV-2 emergence, and 86 residual serum samples collected in the U.S. for hantavirus diagnostics prior to SARS-CoV-2 emergence). These training set samples were tested with the optimized mix-and-read assay and with ELISA against RBD with the same antigen length (spike residues 319-591). The mix-and-read assay signals obtained from single serum dilution varied by 3 orders of magnitude (Fig. 2A). The values were categorized for receiver operating characteristics (ROC) analysis based on presence or absence of prior PCR-confirmed infection (Fig. 2B, C). We decided to prioritize assay specificity and chose the cut-off value of 2.5, which enabled the highest sensitivity (antibodies detected in $92.9 \%$ of the samples with an earlier SARS-CoV-2 nucleic acid finding) while maintaining $100 \%$ specificity. We noticed that the average negative result was somewhat below 1 , indicating modest signal inhibition by serum. The high cut-off value ( $>6$ standard deviations above the average of negative sample signal) also minimizes impact of user error in case sample is omitted. Agreement between ELISA and the mix-and-read assay was very high (kappa value $0.949 ; 95 \%$ confidence interval $0.908-0.989)$. The mix-and-read assay found no antibodies in 6 of 85 samples with 
A)

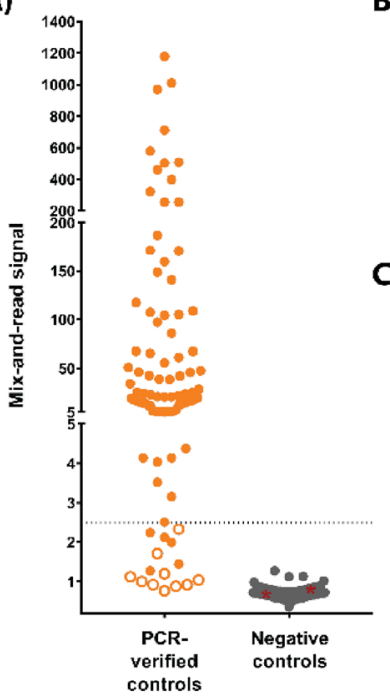

B)

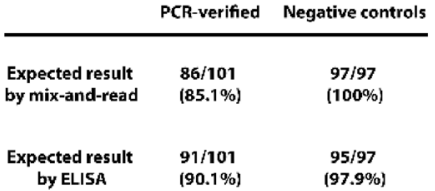

C)

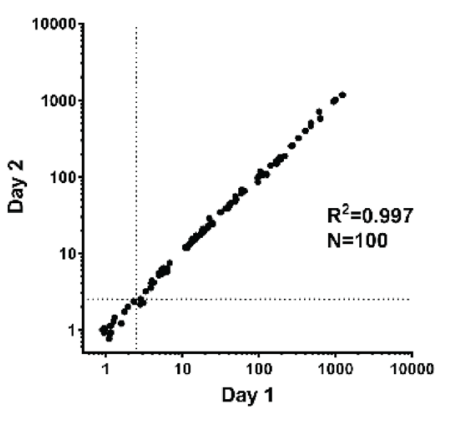

Figure 3. Independent validation set data. (A) Serum samples from confirmed COVID-19 cases and samples considered true negatives were analyzed by RBD ELISA and by the mix-and-read assay using cut-off values determined with the training set. Out of 101 samples with positive SARS-CoV-2 history, 86 were found positive by the mix-and-read assay (orange symbols above signal 2.5), 10 were negative on both assays (open orange symbols), and 5 were positive on ELISA only (orange symbol below 2.5). All negative controls ( $\mathrm{N}=97$, all but one collected before the COVID-19 outbreak) were negative on the mix-and-read assay (gray symbols), while 2 of those samples were positive on ELISA (gray symbols highlighted by red asterisks). (B) Summary of the independent set results. (C) Day-to-day reproducibility of the mix-and-read assay. Samples from the independent validation set were tested on 2 consecutive days and correlation analysis performed on logtransformed values. Samples that gave a discordant qualitative result between the days (near the cut-off, above one day and below the other) were judged negative for purposes of sensitivity and specificity calculations.

confirmed SARS-CoV-2 infection; 4 of these samples were also negative by RBD ELISA; 1 was ELISA positive with a weak signal and 1 was ELISA positive with a moderate signal.

While serum is the standard sample matrix for serology, we wanted to establish whether plasma could be tested as well. Paired human serum and EDTA plasma samples gave similar signals in the mix-and-read assay, suggesting that EDTA plasma samples could also be analyzed (Supplementary Fig. 3A). Furthermore, testing mouse sera from animals immunized with SARS-CoV-2 spike antigens formally showed that the assay works with non-human serum as well (Supplementary Fig. 3B).

Independent validation set. Using the cut-off value established with the training set, we tested 101 more serum samples from individuals with previous confirmed SARS-CoV-2 infection and 97 samples from negative control individuals (96 of which were collected before the outbreak) (Fig. 3A). The RBD ELISA gave a positive result for 91 of 101 positive control samples, while the mix-and-read results were positive for 86 of those samples. Conversely, the mix-and-read assay correctly identified the 97 negative samples, maintaining 100\% specificity, while the RBD ELISA gave negative results for 95 of 97 negative controls ( $97.9 \%$ specificity) (Fig. 3B). The independent set results from the two assays were in high agreement (kappa value $0.929 ; 95 \%$ confidence interval 0.877-0.980). Repeat mix-and-read analysis of 100 samples exhibited high day-to-day signal reproducibility (Fig. 3C), as did 3 positive quantitation controls and a negative control that were used to monitor run-to-run reproducibility (Supplementary Fig. 4). Combined ELISA results and ROC analysis are presented in Supplementary Fig. 5.

Linear range. The assay principle used sets a theoretical upper limit to antibody quantitation. If specific antibodies (or, more precisely, high-affinity antigen binding sites) exceed RBDs, then the number of antibody molecules binding 2 RBDs will decline whereas the number of antibodies binding only 1 or no RBDs will increase. Therefore, increasing the antibody concentration excessively may actually result in declining signal (so called 'hook effect'). To test the theory in practice, monoclonal antibodies were spiked into non-reactive human serum at concentrations between $1 \mathrm{ng} / \mathrm{mL}$ and $100 \mu \mathrm{g} / \mathrm{mL}$. Dose response curves showed that maximal signal was obtained at $\mathrm{mAb}$ concentrations $10-30 \mu \mathrm{g} / \mathrm{mL}$, corresponding to 1:1 to 1:3 stoichiometry between the $\mathrm{RBDs}$ and the antigen binding sites (Fig. 4A). Increasing the $\mathrm{mAb}$ concentration to $100 \mu \mathrm{g} / \mathrm{mL}$ resulted in lower, though strong signal, suggesting that correct qualitative results would be obtained with even the most reactive clinical samples.

Single-dilution quantitation with the mix-and-read assay. Titration results with monoclonal antibodies suggested that the assay signal saturates and declines when excessive antibody concentrations are used. 
A)

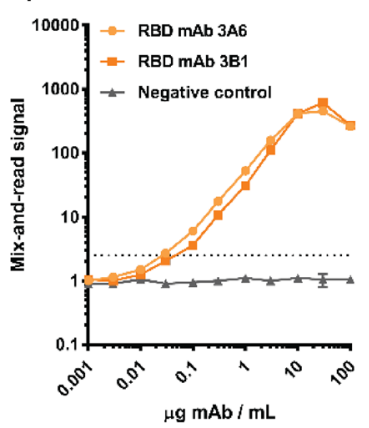

B)

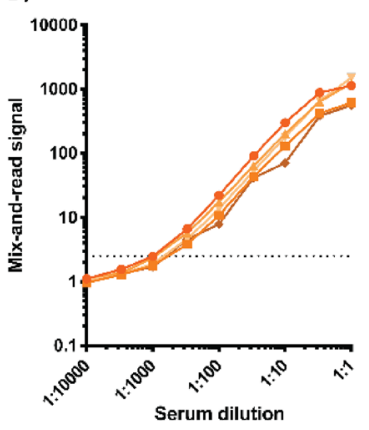

C)

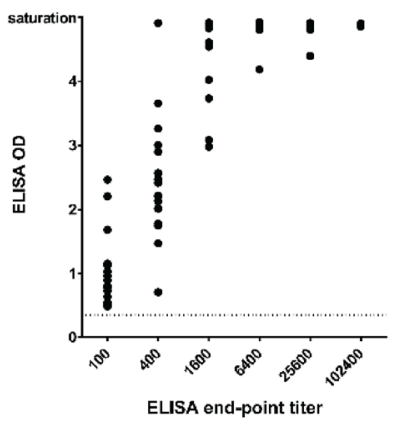

D)

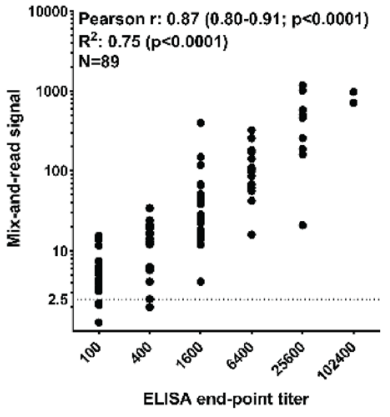

Figure 4. Quantitation. (A) Two positive control IgG $\mathrm{mAbs}$ and a negative control $\mathrm{mAb}$ were titrated to determine the linear range of the mix-and-read signal. (B) Five serum samples that gave a strong signal in initial testing (503-1179 fold over background) were titrated in negative human serum to determine if they reached the saturation level identified by mAbs. (C) Correlation of ELISA end-point titer and ELISA optical density from 1:100 sample dilution. (D) Correlation of ELISA end-point titer and mix-and-read signal. Correlation analysis (Pearson $\mathrm{r}$ and $95 \%$ confidence interval) and linear regression were performed on log-transformed mixand-read data and log2-transformed ELISA end-point titers. (A) and (B): technical duplicates with averages and ranges presented.

To test if the same phenomenon could impact SARS-CoV-2 RBD antibody quantitation in clinical serum samples, 5 samples with high signal-to-background ratios were serially diluted in non-reactive human serum and dose-response curves were generated. All samples gave the strongest signal when undiluted, showing that even the most reactive samples among the $>180$ positives identified here do not contain high enough amounts of specific antibodies to saturate the assay. However, the shape of the dose-response curves near 1:1 dilution suggest that these strong samples are approaching the higher quantitation limit (Fig. 4B).

To investigate if SARS-CoV-2 RBD antibody titers could be inferred from a single screening dilution, endpoint ELISA titers were determined for 89 positive samples of the validation set. Plotting ELISA optical density (OD) values from the screening dilution of 1:100 against the end-point titer showed the expected correlation at low dilutions but loss of quantitation in samples that reached saturation OD in the screening (Fig. 4C). In contrast, no apparent saturation was seen in the mix-and-read assay, and those values and ELISA end-point titers correlated at Pearson $\mathrm{r} 0.87$ (95\% confidence interval $0.80-0.91, p<0.0001)$ and $\mathrm{R}^{2} 0.75(p<0.0001)$, indicating that sample screening at a single dilution is predictive of ELISA endpoint titer in a semi-quantitative manner (Fig. 4D).

\section{Discussion}

With nearly 500 samples analyzed in parallel, our mix-and-read assay results and RBD ELISA results were in excellent agreement. The observed specificity and sensitivity characteristics are comparable to those of independently evaluated, high-performing commercial assays ${ }^{27,28}$, although formal comparisons are best done with standardized validation sample sets.

The mix-and-read assay identified RBD antibodies in $88.7 \%$ of the samples with history of confirmed SARSCoV-2 infection, while our RBD ELISA identified antibodies in $92.5 \%$ of those samples. The time interval from symptom onset to sampling was known for 9 of the 14 samples that were found seronegative by both assays and ranged from 14 to 30 days, suggesting that time of sampling was not the cause of negative results. Since the mixand-read assay can detect IgM antibodies as well as IgG antibodies, class switching should not be required for detecting seroconversion. However, in general, the lower physiological concentration of IgM antibodies may render them the less potent assay activator as compared to IgG antibodies. In conclusion, the implied seroconversion rate of $\sim 90 \%$ is comparable to rates reported by others ${ }^{5,10,29}$, although universal seroconversion has also been reported ${ }^{30}$.

The mix-and-read assay showed $100 \%$ specificity in this sample set while the specificity of ELISA was $98 \%$. The wide dynamic range of the mix-and-read assay allowed us to keep the cut-off value over 6 standard deviations above the mean of the negative controls. Although apparent sensitivity could be marginally improved and the correlation of the two assays further increased by lowering the threshold and accepting false positives at low frequency, we chose to use a cut-off that emphasizes specificity. It is important to remember that, especially in populations in which antibody prevalence is low, the positive predictive value (likelihood that a positive result is correct) deteriorates rapidly when specificity falls below $100 \%{ }^{31,32}$. For example, a test with $99 \%$ specificity and high sensitivity may be useful for many purposes in an area of high prevalence, but in an area where true prevalence is $1 \%$, half of the positive test results would be false. Of note, 2 of the 6 negative control samples that produced an ELISA signal were near the chosen ELISA cut-off, but 4 (collected prior to recognized SARS-CoV-2 emergence) gave moderate to strong OD values, implying cross-reactivity between the RBD antigen and unknown antigens. Interestingly, despite the high overall correlation between the two assays, these ELISA-reactive samples were negative when read on the mix-and-read assay ( 3 with signal $<1$, one being close to the cut-off value 2.5 ). The reason for this phenomenon is unknown, but increased specificity may arise from factors like the strict requirement for simultaneous binding of 2 antigens, better preservation of antigen structure in solution than on plate surface, or steric masking of cross-reactive epitopes by the split luciferase moieties. 
One limitation of our study is that the work was conducted primarily to evaluate a new diagnostic approach. Control samples were sourced based on availability and not as part of a serological survey, and some associated data, including demographics, accurate location, symptoms, underlying conditions, and hospitalization history of the individuals were absent or incomplete and are not reported here. Furthermore, we note that the samples originated in the U.S., and would encourage testing representative control cohorts any time a serological test is implemented in a new area or population, or indeed species, to account for potential differences in baseline reactivity. Of note, we found that convalescent SARS-CoV-1 sera can also activate the assay (data not shown). While this has little practical relevance in human diagnostics due to the small number of convalescent SARSCoV-1 patients, it is noteworthy with regards to ecological investigations and instances where infection with a closely related virus is a possibility.

In summary, we used SARS-CoV-2 as an example to demonstrate that modern protein complementation enables design of simple yet robust serological assays that are easy to automate. Complementation detects antibodies due to their binding of multiple antigens simultaneously, and thus the assay has no apparent antibody class or species restrictions. The improved dynamic range means that more information can be derived from primary screening than is possible with ELISA. Complementation-based serology has the power to increase the output of human SARS-CoV-2 serology efforts and to advance One Health investigations.

\section{Methods}

While this manuscript was in preparation, a qualitative commercial product utilizing the same principle was released (Promega). The two systems have been developed independently and the technical details of the commercial product are unavailable.

Protein expression. Promega NanoBit split luciferase fragments LgBit (large bit) and SmBit (small bit) were fused to the N-terminus of SARS-CoV-2 isolate Wuhan-Hu-1 (GenBank MN908947) RBD. The sequences were separated by a flexible region consisting of a 5-residue linker (GGGGS), $8 \times$ His, and a 16-residue linker (SGSSGGGGSGGGGSSG). IL-6 signal peptide was used to enable secretion. Different RBD lengths, including residues 319-541 and 319-591 of the viral glycoprotein spike were used in the optimization phase. All data presented here are from the 319-591 version of the RBD.

The DNA sequences for the above-described fusion proteins were cloned into an episomal vector (System Biosciences) into which a puromycin resistance cassette had been added to enable selection of pools stably expressing these proteins. To express the proteins, human Expi293 cells (Thermo Scientific), growing in Expi293 Expression medium, were transfected with FectoPRO reagent (PolyPlus Transfection). The supernatants were clarified by slow-speed centrifugation and filtered over polyethersulfone ( 0.2 micron) membranes, and His-tagged proteins were purified by immobilized metal affinity chromatography using HisTrap Excel nickel columns (Cytiva). After dialysis in PBS, the proteins were flash frozen in liquid nitrogen and stored at $-80^{\circ} \mathrm{C}$.

The proteins for RBD/ACE2 binding assay were produced the same way as above. Human codon-optimized ACE2 (residues 1-615, NM_001371415) was expressed with C-terminal LgBit using the same linker and His-tag sequence as described earlier. Similarly, RBD (residues 1-14 and 319-541) was expressed with C-terminal SmBit.

The RBD that was used as ELISA antigen consisted of residues 319-591 and was expressed with an N-terminal IL- 6 signal peptide, $8 \times$ His and 3C protease cleavage site. The protein was produced and His-purified as above, and the epitope tag removed by digestion with $3 \mathrm{C}$ protease. The resulting product was further purified by sizeexclusion chromatography using Superdex 200 columns.

Mix-and-read assay procedure. After initial optimization in a 96-well format, the assay was moved to 384-well plates, and all data presented here were produced using that setup. $20 \mathrm{ng}$ of SmBit-SARS-2-RBD, and equivalent amount of LgBit-SARS-2-RBD in 1:1 molar ratio were added per well of 384-well plates in $20 \mu \mathrm{L} 1 \%$ BSA/PBS (w/V) diluent. $10 \mu \mathrm{L}$ sample was then added, and the mix was incubated for $1 \mathrm{~h}$ at room temperature. $30 \mu \mathrm{L}$ Promega NanoGlo assay reagent was then added and the luminescence resulting from SmBit and LgBit proximity was quantified $10 \mathrm{~min}$ later using a Synergy Neo2 instrument (BioTek) with $0.5 \mathrm{~s}$ integration time and with gain set to 200 and read height to $7.5 \mathrm{~mm}$. Wells with diluent instead of sample were used to determine the assay baseline. Samples with strong signal (up to 2-3 orders of magnitude above baseline) were removed and the plate was read immediately again to minimize error from signal leak between wells. Assay performance was monitored using 4 standards: normal human serum (from plasma) as a negative control, and RBD-specific mouse monoclonal antibody $3 \mathrm{~A} 2$ spiked into the same serum at 3 different concentrations $(10,2.5$, and $0.5 \mu \mathrm{g} /$ $\mathrm{mL})$.

ELISA. ELISAs were conducted against in-house produced SARS-CoV-2 RBD antigen (spike residues 319591). $50 \mathrm{ng}$ of antigen was bound to Immulon $2 \mathrm{HB}$ plates by overnight incubation in $100 \mu \mathrm{L}$ PBS at $4{ }^{\circ} \mathrm{C}$. Wells without antigen were similarly prepared. Wells were washed $2 \times$ with $300 \mu \mathrm{L} 0.1 \%$ Tween-20 in PBS (PBS-T) and blocked with $300 \mu \mathrm{L} 5 \%(\mathrm{w} / \mathrm{V}$ ) non-fat dry milk (CellSignaling Technologies) in PBS-T for $1 \mathrm{~h}$ at room temperature (RT). After 3 washes, samples diluted $1: 100$ in $100 \mu \mathrm{L} 5 \%$ milk in PBS-T were added and incubated for $1 \mathrm{~h}$ at RT. The plates were washed $3 \times$, and mouse secondary anti-human IgG antibody conjugated to HRP (Accurate Chemical \#JMH035098) was added at 1:10.000 dilution in $100 \mu \mathrm{L} 5 \%$ milk in PBS-T and incubated for $30 \mathrm{~min}$ at RT. After 3 washes, $100 \mu \mathrm{L}$ TMB Ultra ELISA substrate (Thermo Fisher Scientific) was added and reactions were stopped after $20 \mathrm{~min}$ at RT with addition of $100 \mu \mathrm{L} 1 \mathrm{M}$ hydrochloric acid. Optical density was read at $450 \mathrm{~nm}$ and densities from antigen-containing wells were corrected by reducing the values obtained from respective no-antigen wells. 
RBD-ACE2 binding assay procedure. $3.2 \mathrm{ng}$ RBD-Smbit (residues 319-541, C-terminal tag) was diluted in $20 \mu \mathrm{L} 0.02 \% \mathrm{BSA} / \mathrm{PBS}$ and mixed with $20 \mu \mathrm{L} \mathrm{mAb}$ dilution $(17 \mu \mathrm{g} / \mathrm{mL}$ ) or $\mathrm{mAb}$ fragment dilution (corresponding molarity) in 96-wells. After $1 \mathrm{~h}$ incubation at RT, ACE2-LgBit was added in volume of $20 \mu \mathrm{L}$ so that $10 \times$ molar excess over the RBD was achieved. After $1 \mathrm{~h}$ incubation at RT, $60 \mu \mathrm{L}$ NanoGlo reagent (Promega) was added and luminescence quantified as with the mix-and-read assay. Wells where antibody dilution was substituted with the diluent served to determine the baseline signal of RBD-ACE2 binding.

Monoclonal antibodies and mouse sera. Generation of monoclonal antibodies and mouse sera is described elsewhere ${ }^{33}$. All animal procedures were approved by the Institutional Animal Care and Use Committee of the Georgia Institute of Technology and performed in accordance with relevant guidelines and regulations.

Monoclonal antibody $3 \mathrm{~A} 2$ was cleaved into $\mathrm{F}\left(\mathrm{ab}^{\prime}\right)_{2}$ and Fab fragments using Pierce Mouse IgG1 Fab and $\mathrm{F}\left(\mathrm{ab}^{\prime}\right) 2$ Preparation Kit (Thermo Fisher Scientific) according to manufacturer's instructions. Briefly, the two fragments were generated by controlling the specificity of immobilized ficin by cysteine- $\mathrm{HCl}$ concentration and incubation time. Immobilized protein $\mathrm{A}$ was used to remove uncleaved $\mathrm{mAb}$ and $\mathrm{Fc}$ fragments. The fragments were concentrated with $500 \times$ buffer exchange against PBS using spin columns with $10 \mathrm{kDa}$ molecular weight cut-off (Amicon, Millipore Sigma). mAbs were diluted to $10 / 3 / 1 / 0.3 \mu \mathrm{g} / \mathrm{mL}$ and the fragments were diluted to corresponding antigen-binding molarities and analyzed as above.

$\mathrm{mAb} 3 \mathrm{~A} 2$ was also used as quantification control. For this purpose, purified $\mathrm{mAb}$ was spiked into normal human serum at concentrations of $10,2.5$, or $0.5 \mu \mathrm{g} / \mathrm{mL}$; normal serum was left unspiked as the negative control. The preparations were aliquoted and frozen, and freshly thawed aliquots were used to monitor run-to-run and day-to-day assay performance.

Samples. Positive and negative human serum and plasma samples were sourced via BioIVT, iSpecimen, StemExpress and Emory University. Time to collection from symptom onset was available for 129 of 186 positive serum controls and varied from 6 to 71 days, with $10 \%$ and $90 \%$ percentiles at 15 and 53 days, respectively. Additionally, residual serum samples collected for hantavirus and Zika virus serology prior to emergence of SARS-CoV-2 were tested as negative controls (as part of the training set and the validation set, respectively). The hantavirus samples were inactivated by $5 \times 10^{6} \mathrm{RAD}$ gamma irradiation from a ${ }^{60} \mathrm{Co}$ source. All samples were heated to $56^{\circ} \mathrm{C}$ for $10 \mathrm{~min}$ before use. The serum samples from commercial sources were analyzed in a blinded fashion. Approximately $6 \%$ of initially analyzed samples were excluded from final analysis after identifying multiple samples from the same donor (pre-outbreak residual samples) or due to inconclusive information regarding previous SARS-CoV-2 nucleic acid testing results (commercially sourced samples after de-blinding).

Ethics statement. This study did not involve contact with patients, and no specimens were collected specifically for the purposes of this study. Residual samples were used for diagnostics development under a protocol that was reviewed and approved by the Centers for Disease Control and Prevention Institutional Review Board with an approved waiver of informed consent (See 45 C.F.R. part 46; 21 C.F.R. part 56). All methods involving human biological samples were carried out in accordance with relevant guidelines and regulations.

Purification of IgG and IgM from human serum. Human serum samples from SARS-CoV-2 positive donors or control donors ( $\mathrm{N}=14$ each) were pooled, and IgG and IgM antibodies were purified according to manufacturer's recommendations using CaptureSelect $\mathrm{CH} 1-\mathrm{Xl}$ and Poros CaptureSelect IgM Affinity matrices (Thermo Fisher Scientific). The samples were concentrated and the buffer was changed to PBS using $10.000 \mathrm{Da}$ spin columns (Amicon). The purified fractions were analyzed by SDS-PAGE both under non-heated, nonreduced conditions and under heated, reduced conditions.

Statistics. Statistical analyses were performed using GraphPad Prism software version 7.04 except for the kappa statistic, which was determined using the online version of GraphPad at https://www.graphpad.com/ quickcalcs/kappa1.cfm (confidence interval calculated with equations by Fleiss).

\section{Data availability}

Unique research materials will be shared upon completion of appropriate material transfer agreements.

Received: 2 October 2020; Accepted: 18 May 2021

Published online: 10 June 2021

\section{References}

1. Lerner, A. M. et al. The COVID-19 serology studies workshop: recommendations and challenges. Immunity 53, 1-5 (2020).

2. Wu, F. et al. A new coronavirus associated with human respiratory disease in China. Nature 579, 265-269 (2020)

3. WHO. WHO Statement Regarding Cluster of Pneumonia Cases In Wuhan, China. Available at: https://www.who.int/china/news/ detail/09-01-2020-who-statement-regarding-cluster-of-pneumonia-cases-in-wuhan-china (2020).

4. Yong, S. E. F. et al. Connecting clusters of COVID-19: an epidemiological and serological investigation. Lancet Infect. Dis. 20, 809-815 (2020).

5. Long, Q.-X. et al. Clinical and immunological assessment of asymptomatic SARS-CoV-2 infections. Nat. Med. 26, 1200-1204 (2020).

6. Ibarrondo, F. J. et al. Rapid decay of anti-SARS-CoV-2 antibodies in persons with mild Covid-19. N. Engl. J. Med. 383, 1085-1087 (2020).

7. Perreault, J. et al. Longitudinal analysis of the humoral response to SARS-CoV-2 spike RBD in convalescent plasma donors. bioRxiv. https://doi.org/10.1101/2020.07.16.206847 (2020) 
8. Callow, K. A., Parry, H. F., Sergeant, M. \& Tyrrell, D. A. J. The time course of the immune response to experimental coronavirus infection of man. Epidemiol. Infect. 105, 435-446 (1990).

9. Reed, S. E. The behaviour of recent isolates of human respiratory coronavirus in vitro and in volunteers: evidence of heterogeneity among 229E-related strains. J. Med. Virol. 13, 179-192 (1984).

10. Staines, H. M. et al. Dynamics of IgG seroconversion and pathophysiology of COVID-19 infections. medRxiv. https://doi.org/10. $1101 / 2020.06 .07 .20124636(2020)$

11. Wajnberg, A. et al. SARS-CoV-2 infection induces robust, neutralizing antibody responses that are stable for at least three months. medRxiv. https://doi.org/10.1101/2020.07.14.20151126 (2020)

12. Rodda, L. B. et al. Functional SARS-CoV-2-specific immune memory persists after mild COVID-19. medRxiv. https://doi.org/10. $1101 / 2020.08 .11 .20171843(2020)$

13. Edridge, A. W. D. et al. Coronavirus protective immunity is short-lasting. medRxiv. https://doi.org/10.1101/2020.05.11.20086439 (2020)

14. Shi, J. et al. Susceptibility of ferrets, cats, dogs, and other domesticated animals to SARS-coronavirus 2. Science 368, 1016-1020 (2020).

15. Fagre, A. et al. SARS-CoV-2 infection, neuropathogenesis and transmission among deer mice: implications for reverse zoonosis to New World rodents. bioRxiv. https://doi.org/10.1101/2020.08.07.241810 (2020)

16. Schlottau, K. et al. SARS-CoV-2 in fruit bats, ferrets, pigs, and chickens: an experimental transmission study. Lancet Microbe https://doi.org/10.1016/S2666-5247(20)30089-6 (2020).

17. Chan, J.F.-W. et al. Simulation of the clinical and pathological manifestations of Coronavirus Disease 2019 (COVID-19) in golden Syrian hamster model: implications for disease pathogenesis and transmissibility. Clin. Infect. Dis. https://doi.org/10.1093/cid/ ciaa325 (2020).

18. Sia, S. F. et al. Pathogenesis and transmission of SARS-CoV-2 in golden hamsters. Nature 583, 834-838 (2020).

19. Oreshkova, N. et al. SARS-CoV-2 infection in farmed minks, the Netherlands, April and May 2020. Euro Surveill. https://doi.org/ 10.2807/1560-7917.ES.2020.25.23.2001005 (2020).

20. Wouters, E., Vasudevan, L., Crans, R. A. J., Saini, D. K. \& Stove, C. P. Luminescence- and fluorescence-based complementation assays to screen for GPCR oligomerization: current state of the art. Int. J. Mol. Sci. 20, 1-35 (2019).

21. Li, P., Wang, L. \& Di, L. Applications of protein fragment complementation assays for analyzing biomolecular interactions and biochemical networks in living cells. J. Proteome. Res. 18, 2987-2998 (2019).

22. Dixon, A. S. et al. NanoLuc complementation reporter optimized for accurate measurement of protein interactions in cells. ACS Chem. Biol. 11, 400-408 (2016).

23. Okba, N. M. A. et al. Severe acute respiratory syndrome coronavirus 2-specific antibody responses in coronavirus disease patients. Emerg. Infect. Dis. 26, 1478-1488 (2020).

24. Premkumar, L. et al. The RBD of the spike protein of SARS-group coronaviruses is a highly specific target of SARS-CoV-2 antibodies but not other pathogenic human and animal Coronavirus antibodies. medRxiv. https://doi.org/10.1101/2020.05.06.20093377 (2020)

25. Wrapp, D. et al. Cryo-EM structure of the 2019-nCoV spike in the prefusion conformation. Science 367, 1260-1263 (2020).

26. Amanat, F. et al. A serological assay to detect SARS-CoV-2 seroconversion in humans. Nat. Med. 26, 1033-1036 (2020).

27. Public Health England. COVID-19: Laboratory Evaluations of Serological Assays-GOV.UK. Available at: https://www.gov.uk/gover nment/publications/covid-19-laboratory-evaluations-of-serological-assays. (Accessed 11th August 2020)

28. Brochot, E. et al. Comparison of different serological assays for SARS-CoV-2 in real life. J. Clin. Virol. 130, 104569 (2020).

29. Payne, D. C. et al. SARS-CoV-2 infections and serologic responses from a sample of U.S. Navy service members-USS Theodore Roosevelt, April 2020. Morb. Mortal. Wkly. Rep. 69, 714-721 (2020).

30. Long, Q. X. et al. Antibody responses to SARS-CoV-2 in patients with COVID-19. Nat. Med. 26, 845-848 (2020).

31. Kumleben, N. et al. Test, test, test for COVID-19 antibodies: the importance of sensitivity, specificity and predictive powers. Public Health 185, 88-90 (2020).

32. Brownstein, N. C. \& Chen, Y. A. Are antibodies tests accurate? Understanding predictive values and uncertainty of serology tests for the novel coronavirus. medRxiv. https://doi.org/10.1101/2020.06.04.20122358 (2020)

33. Chapman, A. P. et al. Rapid development of neutralizing and diagnostic SARS-COV-2 mouse monoclonal antibodies. bioRxiv. https://doi.org/10.1101/2020.10.13.338095 (2020)

\section{Acknowledgements}

We thank Tatyana Klimova for assistance in editing this manuscript; John Klena, Inna Krapiunaya and Jarad Schiffer for assistance with sample access and the Laboratory Task Force of the CDC COVID-19 response for their critical project review and resource support. This research was made possible using samples obtained from the CDC Biorepository. The findings and conclusions in this report are those of the authors and do not necessarily represent the official position of the Centers for Disease Control and Prevention.

\section{Author contributions}

M.H.K. and E.B. designed and conducted experiments and contributed to the manuscript; P.C. and H.M.J. conducted experiments; A.P.C., J.L., A.C., X.T., R.E.W., K.B.M., M.G.F. and J.M.G. developed monoclonal antibodies and mouse sera; M.P., A.J.S., P.S.S., J.M.C. and S.M.O. acquired samples and compiled associated data; T.D.F. reviewed statistics; L.K.M., M.F., J.M.M. and C.F.S. managed the project and contributed to the manuscript. All authors reviewed the manuscript.

\section{Funding}

This work was funded by the Centers for Disease Control and Prevention.

\section{Competing interests}

The authors declare no competing interests.

\section{Additional information}

Supplementary Information The online version contains supplementary material available at https://doi.org/ 10.1038/s41598-021-91300-5.

Correspondence and requests for materials should be addressed to C.F.S.

Reprints and permissions information is available at www.nature.com/reprints. 
Publisher's note Springer Nature remains neutral with regard to jurisdictional claims in published maps and institutional affiliations.

(c) (i) Open Access This article is licensed under a Creative Commons Attribution 4.0 International License, which permits use, sharing, adaptation, distribution and reproduction in any medium or format, as long as you give appropriate credit to the original author(s) and the source, provide a link to the Creative Commons licence, and indicate if changes were made. The images or other third party material in this article are included in the article's Creative Commons licence, unless indicated otherwise in a credit line to the material. If material is not included in the article's Creative Commons licence and your intended use is not permitted by statutory regulation or exceeds the permitted use, you will need to obtain permission directly from the copyright holder. To view a copy of this licence, visit http://creativecommons.org/licenses/by/4.0/.

This is a U.S. Government work and not under copyright protection in the US; foreign copyright protection may apply 2021 